\title{
Myomectomy: TU Teaching Hospital experiences
}

\author{
Geeta Gurung, Neelam Pradhan, Suniti Rawal, \\ Ashma Rana, Seeta Ghimire, Josie Baral \\ Dept of Obs/Gyn TUTH, Kathmandu Nepal
}

\begin{abstract}
Aim: To study, open myomectomy operation for uterine myoma/s as of present day practices in women of reproductive age attending TU Teaching Hospital.

Methods: This is a retrospective study where we reviewed the medical records of myomectomy cases from 2060-2065(2003 April-2009 April).

Result: Total 40 cases of myomectomy performed in TU Teaching hospital for myoma size varying from $\geq 10$ weeks (5), 12-16 weeks (15), 17-22 weeks (5), 23-28 weeks (5) and unknown (2) in women of reproductive age $\left[30-34(16), \geq 29\right.$ (13), 35-39 (9), 40- 44 (1) and $\geq 45$ (1)] in 13 (unmarried); nullipara (17); $P_{1-2}$ (15) $P_{3.4}$ (1) for reasons of infertility(12), dysmenorrhea (12), menorrhagia (8), urinary problems( 7 ) abdominal pain (3).

At caesarean, myomectomy has been done in special circumstances for subbersous pedunculated myoma and broad ligament myoma, one each.

Solitary myomas were 29 and multiple myomas were 8; a case each of broad ligament and cervical myoma (1), was later removed vaginally. Rests had abdominal procedure and was facilitated by Bonney's hood incision and posterior incision 8 each, anterior 7, fundal 2, using multiple incision technique in 11 along with secondary tunneling incision (3). Uterine cavity was opened in 3 resulting in blood loss up to $\geq 500 \mathrm{ml}$ in 2. Degeneration were noticed in 11 [cystic (4), fatty (2), calcification (2) red (2) and myxoid (1)] and none with post operative morbidity. Pregnancies were recorded in 3 cases.

Conclusion: Open abdominal myomectomy, for uterine myoma/s has been a recognized procedure for infertile women in the past; but in view of less morbidity as shown by this present study myomectomy can be safely practiced in women desiring uterine preservation besides infertility care.
\end{abstract}

Key words: Degeneration, dysmenorrhoea, menorrhagia, myomectomy

\section{Introduction}

Uterine myomas are the most common benign tumors of the female reproductive tract. They are found in 25 $35 \%$ of women over 35 and are also common towards the end of the reproductive years. ${ }^{1}$ Generally speaking, only $50 \%$ of the myomas cause symptoms, like abnormal vaginal bleeding (menorrhagia), pressure symptoms, infertility etc. requiring some form of surgery. Myomectomy, one of the conservative operative approaches deals with the removal of myoma alone with the uterine preservation. This article concentrates on myomectomy performed for various types of myoma arising from genital tract, in women of reproductive age groups.

\section{Methods}

This is a retrospective study where medical records of myomectomy cases were reviewed from 2060-2065 (2003 April-2009 April). The IP number were retrieved from OT register, female surgical ward and post operative ward record that finally facilitated collection of charts from medical record section. 


\section{Result}

Total 40 cases of myomectomy (fig 1-4), which were performed during the study period, were analyzed. Age group of the women ranged from 30-34 years (16), $\leq 29$ years (13), 35-39 years (9), 40- 44 years (1) and $\geq 45$ years (1). Of them 13 were unmarried women. Majorities of the women were of low parity: nullipara (17), $\mathrm{P}_{1-2}$ (15), $\mathrm{P}_{3-4}(1)$. Infertility and dysmenorrhea were the most common symptoms (12 each), followed by mass abdomen (9), menorrhagia (8), urinary problems 7 [difficulty in passing urine (4), increase frequency of micturation (3) and acute retention on urine (1)]; Pain abdomen 3 (one with myxoid degeneration), irregular cycle 2, heaviness in the abdmen (1). However accidental USG detection of myoma was found in two cases and incidental detection of myoma during emergency caesarean for cord prolapse was in one case, which was a case of broad ligament myoma.

$>\quad$ Preoperative uterine size was: $\leq 10$ weeks (5), 1216 weeks (15), >16-22 weeks (5), >22-28 weeks (5) and size not mentioned were (2).

$>\quad$ Of the total cases of myoma, intra mural myoma were the most common type (26), followed by subserous (7), submucous (1), cervical (1) and broad ligament (1).

> Solitary myoma were 29 and the information was available in 25[intramural (22) subserous (3) and sub mucous (0)].

$>\quad$ Of the 8 cases of multiple myomas; maximum numbers of myoma was found in a case which amounted to be 21. Among the eight cases of multiple myoma/s: combination of subserous plus subserous occurred in (2), followed by intramural and intramural (1) and lastly intramural and subserous (1).

> Myomectomy was successfully done via vaginal approach for a case of a cervical myoma. Rests of the cases were done abdominally. Facilitated removal of myoma was undertaken by various incisions Bonney's hood (8); anterior incision (7), posterior incision (8), fundal incision (2).

Multiple incisions were inflicted in 11[three had secondary tunneling incision] (table1).

Table1. Showing the different incisions during myomectomy

\begin{tabular}{lr} 
Bonney's hood & 8 \\
Posterior incision & 8 \\
Anterior incision & 7 \\
Multiple incisions & 11 \\
Secondary tunneling & 3 \\
Caesarean myomectomy & 2 \\
Cavity opened & 3 \\
Vaginal myomectomy & 1 \\
\hline
\end{tabular}

Cavity was opened during surgery in 3 cases. And the estimated blood loss during surgery is as follows: $\leq 300 \mathrm{ml} 17$ [ $(\leq 100 \mathrm{ml}$ in 7 cases $)$; $>100-200 \mathrm{ml}$ in 5 cases; $>200-300 \mathrm{ml}$ in 5 cases], $>300-500 \mathrm{ml}$ in 3 cases and only one case had $>500-700 \mathrm{ml}$ blood loss.

Cut section of 11 cases myoma exhibited different types of degeneration [cystic (4); fatty (2); calcification (2); red degeneration (2) and myxoid degeneration (1).

Following myomectomy, so far three pregnancies have resulted favorably with good neonatal outcome in one woman. An alive healthy baby boy weighing $2800 \mathrm{gm}$ at 37 completed weeks was delivered by caesarean. In the second case, unfortunately pregnancy was interrupted at about 16 weeks due to severe pyelonephritis and the third is being currently followed at antenatal clinic is running third trimester.

\section{Discussion}

Myomectomy was a feared practice in older days and thus was limited in women for reasons of fertility. Currently because of the safety of surgical procedure, improvised technicality, safe anaesthesia, broad spectrum antibiotic coverage, and blood transfusion facilty, myomectomy can be desired surgical modality even in multiparous women; for reasons beyond fertility that is for women's satisfaction of menstrual function. This is because of our observation findings of myoma that have been implicated in younger age women, nullipara or women with low parity. As pointed out from different studies the myomas are the tumors that arise essentially in reproductive age below 35 , this necessitates removal of tumor alone leaving behind the uterus. ${ }^{2,3}$

It has been often questioned, as why to perform myomectomy in women aged around 40. While the explanation can be provided in consideration of the increasing age of menopause at or around 52; rendering satisfaction of menstruation and feeling of youth for a period of a decade. Our study has not shown any serious intra/ postoperative morbidity which again emphasizes on this specialized surgery. May be because of the improvised techniques such as intermittent use of tourniquet, use of misoprostol half an hour pre operatively, incising the myoma near its base to facilitate shorter and easy shelling with minimization of blood loss and operating time, application of better suture materials and uterine closure using baseball suture. ${ }^{4}$

However a chance of recurrence of myoma must be perceived in all these young women. Studies have shown that all the myoma above 10 weeks uterine size or multiple myomas have predisposition for recurrence. 5 


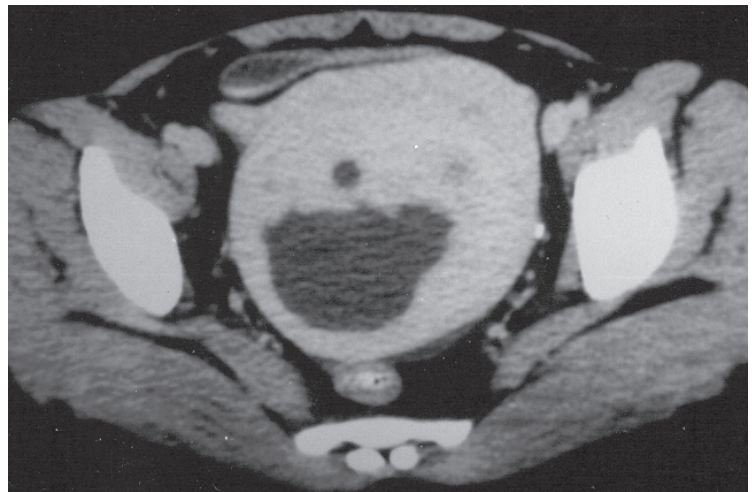

fig1. myoma with degeneration

This statement is more favorable in our situation; as the number multiple myoma in our study is comparatively less.

Pregnancy after myomectomy is possible yet very few of them are recorded. Many women in our society fear

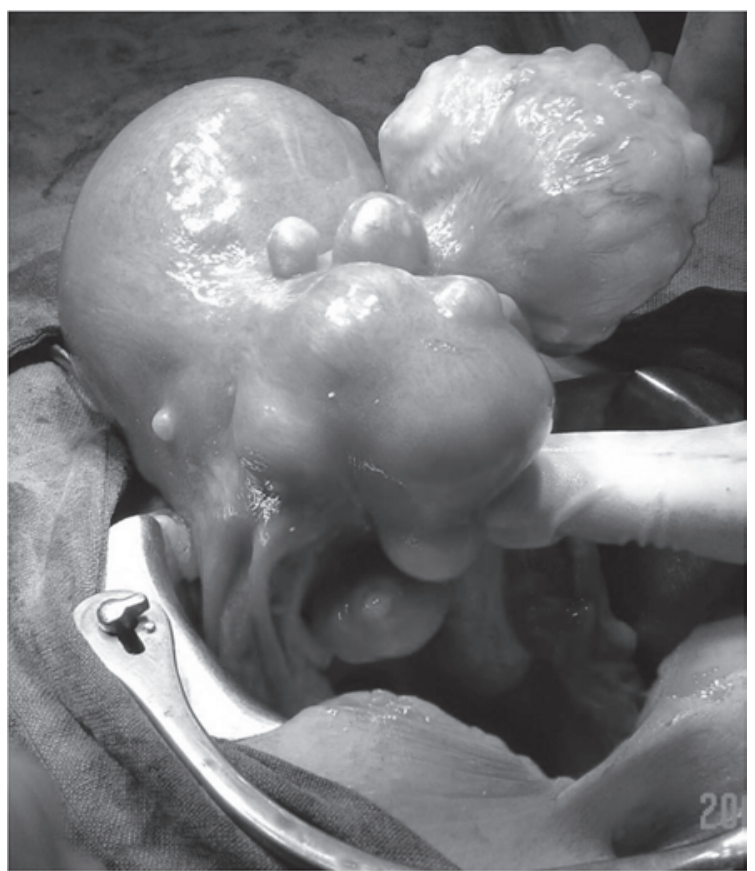

fig 2a. myoma

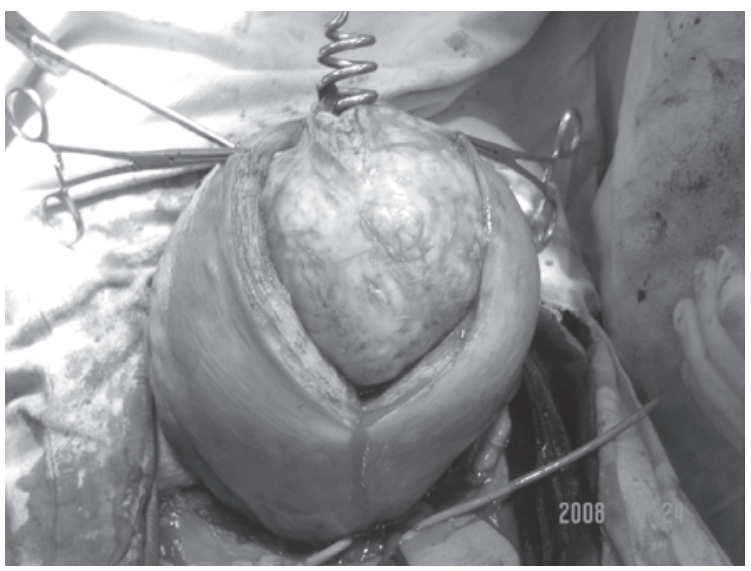

fig 3. single solitary myoma cohabitation in the immediate post operative period and afterwards. Post myomectomy conception and fruitful pregnancy has not been very obliging in our study, maybe because of the involution of uterus was inadequate in cases of larger myoma.

Reduction in uterine size after surgery has been explained to be from the reduction of estrogen receptor lying around the vicinity of myoma. ${ }^{6}$ However failure to involute with persistently enlarged uterine size was seen to be associated with abortion in one post myomecty case.

Yet the presenting symptoms for which the women underwent myomectomy operation subsided or improved tremendously. ${ }^{1}$ Natural conception was possible and so was the symptomatic benefit in the alleged study.

Most surgeons have complied myomectomy in the age 31-40 years. ${ }^{3}$ where as we have done few cases in

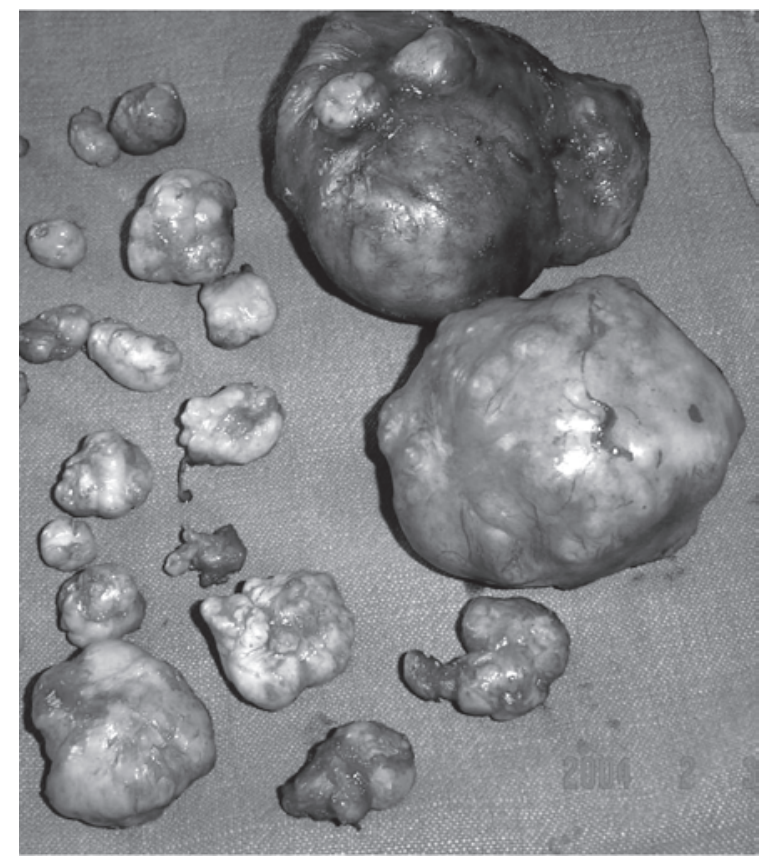

Fig 2b. removal of myoma

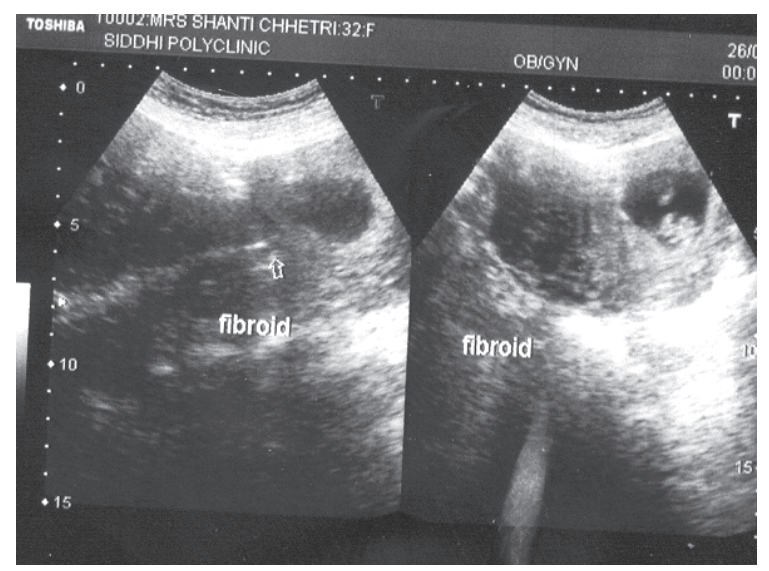

fig 4a. myoma and pregnancy 


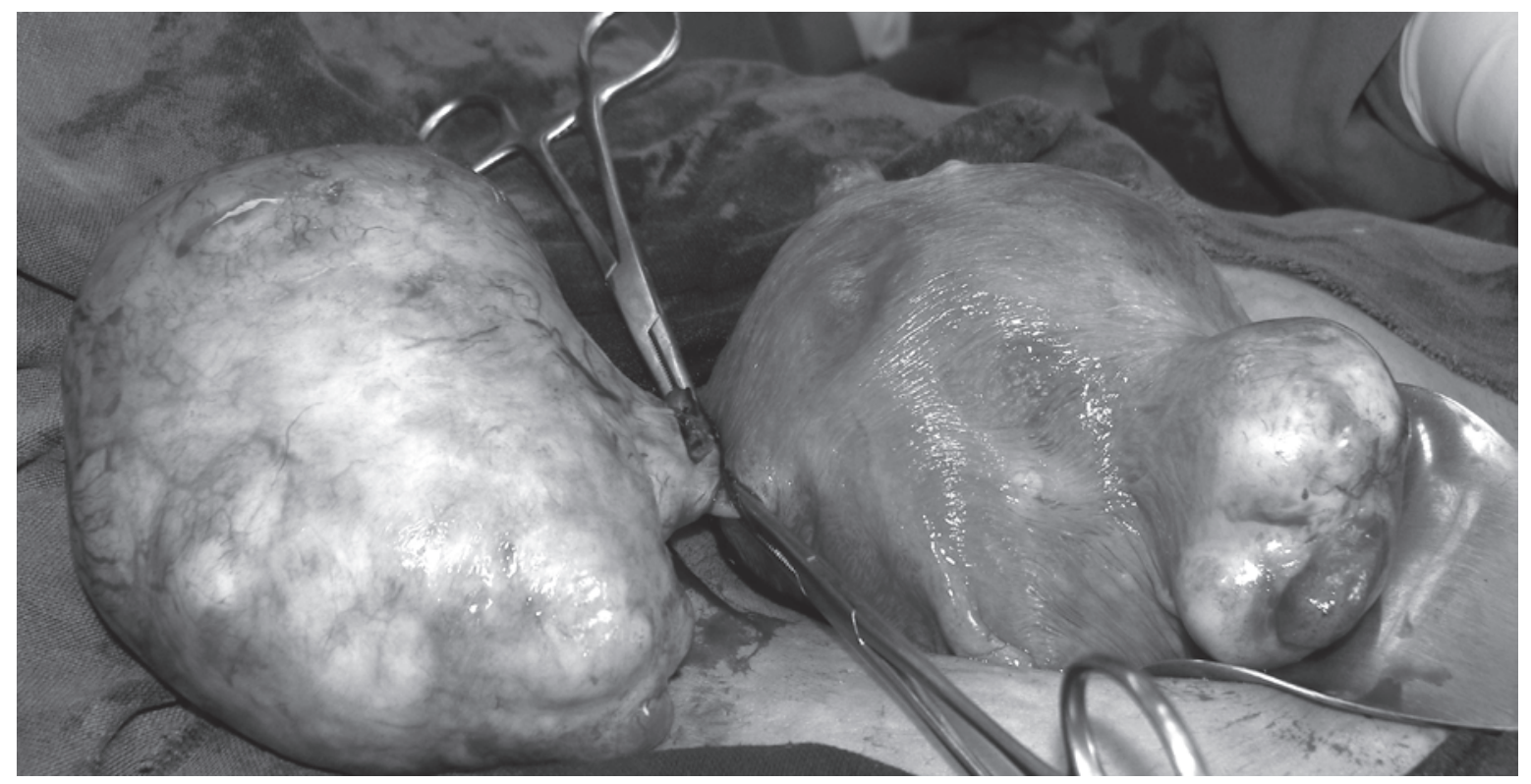

fig 4b. myomectomy for subserous myoma at CS

perimenopausal age for solitary tumors and in view of reducing morbidity associated with extensive surgery. It is noted that intraopeartive blood loss and febrile morbidity following myomectomy have been considerably reduced than hysterectomy. ${ }^{7}$

Therefore the number of myomectomy has seen in increasing trend pursuing a fruitful pregnancy up to three times in a study. ${ }^{8}$ This appears to be additional benefit of myomectomy.

\section{Conclusion}

Open abdominal myomectomy, for uterine myoma/s has been a recognized procedure for infertile women in the past; but in view of less morbidity as shown by this present study myomectomy can be safely practiced in women desiring uterine preservation besides infertility care.

\section{References}

1. Olufowobi O, Sharif K, Papaionnou S, Neelkantan D, Mohammed H, Afnan M. Are the anticipated benefits of myomectomy achieved in women of reproductive age? A 5 year review of the results at a UK tertiary hospital. J Obstet Gynaecol 2004; 24(4):434-40.
2. Aboyeji AP, Ijaiya MA.Uterine fibroids: A tenyear clinical review in Ilorin, Nigeria. Niger J Med. 2002 Jan-March; 11 (1): 16-9.

3. Omu AE, Ihejerica IJ, Tabowei G. Management of uterine fibroids at the University of Benin Teaching Hospital. Trop Doct 1984 Apr; 14 (2): 82-5.

4. Rana A, Gurung G, Amatya A, Khatry R. A simple new technique of performing myomectomy for a predominantly subserosal myoma quickly within few minutes. KUMJ 2007;5(1):110-1

5. Hanafi M. Predictors of leiomyoma recurrence after myomectomy. Obstet Gynecol 2005; 105(4): 877-81

6. John DT, John AR. Liomyomata Uteri and Myomectomy. In: Te Lende's operative gynecology, eighth edition,Lippincott-Raven, Philadelphia 1996: 731- 65.

7. Mohammed NB, NoorAli R, Anandkumar C. Uterine fibroid: Clinical presentation and relative morbidity of abdominal myomectomy and total abdominal hysterectomy in a teaching hospital of Karachi, Pakistan. Singapore Med J 2002; 43(6): 285-95.

8. Gavai M, Hupuczi P, Papp Z. Abdominal myomectomy as an alternative to hysterectomy: analysis of 504 cases. Orv Hetil 2006; 147 (21): 971-8. 